\title{
Physiotherapy protocol with interferential current in the treatment of vaginismus - Observational and prospective study
}

\author{
Carla Maria de Abreu Pereira', Ricardo Thiago Paniza Ambrosio², Erica Maximiano Costa Mantelli Borges³, \\ Sônia Maria Rolim Rosa Lima', Vera Lúcia dos Santos Alves ${ }^{1}$
}

\begin{abstract}
Introduction: Vaginismus is characterized by involuntary recurrent or persistent contraction of the perineum muscles adjacent to the lower third of the vagina when vaginal penetration with penis, finger, tampon or speculum is attempted. However, there may be low adherence to the therapy due to the need of the touch of the perineal region by the therapist. The interferential current can be an alternative, once it can be used externally and superficially. Objective: To evaluate a clinical protocol with the use of interferential current to treat vaginismus and to observe the decrease of pain and the behavior of the electrical activity on the perineal musculature. Methods: The protocol was registered in the Clinical Trials (NCT03176069) and it was approved by the Research Ethics Committee (CAAE: 51995515.4.0000.5479). This will be a controlled clinical trial with a group of patients with Vaginismus $(n=30)$ and another without $(n=30)$. All women will be submitted to evaluation and reassessment with algometry, electromyographic biofeedback and visual analogue pain scale. The vaginismus group will perform 10 sessions, twice a week, with the interferential current (frequency $=2000 \mathrm{~Hz}$, modulated amplitude $=80 \mathrm{~Hz}$, pulse width $=200 \mu$ s and intensity according to sensory level) for 30 minutes. The evolution of vaginismus group before and after the intervention and the correlations between groups will be statistically analysed with the $t$ Student's or the Mann-Whitney test with a significance level of $5 \%(p \leq 0.05)$. Hypothesis: This protocol will present a possible alternative for the treatment of vaginismus and it will investigate the electrical activity of the pelvic floor musculature in vaginismus compared to women who do not suffer from this disorder. It is expected to decrease the pain, to measure the muscular electrical activity before and after the intervention in the vaginismus group and to investigate the muscular electrical activity in the group without sexual dysfunction.
\end{abstract}

Keywords: Vaginismus; Muscle Tone; Sexual Pain; Sexual Dysfunction; Physical Therapy.

\section{INTRODUCTION}

Vaginismus refers to involuntary recurrent or persistent contraction of the perineum muscles adjacent to the lower third of the vagina when penetration is attempted. This generates pain and the sensation prevents the relaxation for penetration of any object - the penis, a tampon or a speculum ${ }^{1-4}$. In the Diagnostic and Statistical Manual of Mental Disorders (DSM-5) ${ }^{5}$, vaginismus is part of the Disorders of Pelvic Pain / Penetration and it can be presented as a disease that arises throughout life. What means that it has always been present since the first sexual experience or it has been acquired. In the International Classification of Diseases (ICD-11), approved by the World Health Organization in 2018 and coming into force in January 2022 , the disease is a sexual disorder of the pain penetration $\left(\right.$ HA20.Z) ${ }^{6}$. Despite the lack of epidemiological studies that define the prevalence of vaginismus, it is clinically estimated between $5-20 \%^{1,2}$. However, it is known that many women do not look for medical treatment because of fear, lack of confidence, discomfort and lack of knowledge. According to this perspective, the population affected by vaginismus is probably underestimated ${ }^{6-8}$

The pain sensation is common to these patients and it can be understood by the different mechanisms, types of neurotransmitters and structures of the central nervous system (CNS) and peripheral system (SNP). Pain can be assessed using features such as the visual analogue scale (VAS) ${ }^{7}$, or by a direct measurement performed by a pressure algometer ${ }^{8}$. The pain symptom that arises under the threat of penetration promotes involuntary contraction that may alter muscle electrical activity. Integral, tonic and contractile musculature allows better vaginal sensitivity and it is essential for sexual activity ${ }^{9}$. Perineal electromyographic biofeedback assists in the evaluation of the perineal musculature and verifies muscle activity $^{10}$.

Among the various techniques and resources of treatment, transcutaneous electrical nerve stimulation (TENS) is a non-pharmacological method used for muscle relaxation

\footnotetext{
Correspondence: Vera Lúcia dos Santos Alves. Address: Rua Dr. Cesário Motta Júnior, 112. Vila Buarque - São Paulo (SP) - Brasil. CEP: 01221-020. Telephone Number: (+55 11) 3872-1966. Email: fisioterapiasc@uol.com.br

1 Faculdade de Ciências Médicas da Santa Casa de São Paulo - São Paulo - SP - Brasil

Full list of author information is available at the end of the article.
}

Financial Support: nothing to declare.

Submission date 07 May 2019; Acceptance date 20 May 2020; Publication date 27 May 2020 
and pain reduction in vaginismus. Devices of interferential current (IC) can be used. Their frequency can vary from $1 \mathrm{~Hz}$ to $4500 \mathrm{~Hz}^{11}$. The interferential current produces low impedance to the skin and it penetrates deeper into the tissue, reduces pain and promotes muscle relaxation ${ }^{12,13}$.

The literature presents some studies that confirm the importance and value of the results achieved in the treatment of vaginismus using physical therapy. Techniques are used for the rehabilitation of sexual dysfunction with the use of manual resources, perineal exercises and dilators. However, low adherence to the treatments is observed, once the touch in the region of the vagina is necessary. There is also the need for a large number of sessions to observe results ${ }^{14-17}$. No studies were found that investigated or associated perineal electromyography algometer and biofeedback with women with vaginismus, nor studies that used the interferential current as a treatment option for these patients. The present study hypothesizes that the reduction of the number of sessions to 10 , as well as an external manipulation of the pelvic floor may positively impact the adherence to the treatment, and that the electromyographic evaluation of the pelvic floor of women with vaginismus has a different muscular electrical activity behavior than the one of healthy women. This abnormal muscular electric activity is probably associated with the evaluation of the pain given by the algometer. The use of interferential current in vaginismus can further expand the possibilities of treatment for the improvement of pain and muscle relaxation.

The objective of this study is to compare the electromyography results of the vagina of women with vaginismus pre and post-treatment evaluation and to test if the interferential current can reduce pain and modify the muscle contractions, observed by means of the examination of the pelvic floor musculature. The hypothesis is that the electromyographic evaluation of the pelvic floor of women with vaginismus can demonstrate a differentiated behavior of the muscular electrical activity, which is associated with the objective evaluation of the pain given by the algometer. The use of interferential current in vaginismus can further expand the possibilities of treatment for the improvement of pain and muscle relaxation.

\section{METHODS}

The protocol was registered in the Clinical Trials (NCT03176069) and it was approved by the Research Ethics Committee (CAAE: 51995515.4.0000.5479).

\section{Recruitment and Sample Selection}

Patients will be evaluated accompanied by the by the Department of Gynecology and Obstetrics of Santa casa de São Paulo, with and without diagnosis of vaginismus.

\section{Inclusion Criteria}

- Age $\geq 18$ and $\leq 45$;

- Nulliparous;

- Heterosexuals;

- Stable relationship for at least six months;

- Sexual activity;

\section{Exclusion Criteria}

- Severe psychiatric disorder, cognitive relegation;

- Vaginismus requiring surgical treatment;

- Partner with sexual dysfunction that prevents penetration;

- Presence of pelvic organ prolapse.

\section{Sample characterization}

The sample size was calculated for the pain symptom with the Central Limit Theorem supported by three studies conducted by Bortolami et al. ${ }^{18}$, Pacik ${ }^{19}$ and Fugl-Meyer et al. ${ }^{20}$. The calculation indicated the need for inclusion of 60 women, 30 with diagnosis of vaginismus (GV - convenience sample) and 30 without vaginismus (GWV). After the selection of the patients and the signing of the consent form, the evaluations will be performed by the physiotherapist, who will perform the allocation of the participants (Figure 1).

\section{Evaluation and Follow-up}

The protocol evaluation and application process will be conducted by a physiotherapist with experience in the evaluation and treatment procedures, who will not know about the objectives of the study. The study timeline will be conducted as follows: algometry evaluation, electromyographic biofeedback and visual analogue scale for pain. The Vaginismus Group (VG) will initiate the protocol two days after the evaluation and will receive IC for 10 sessions performed twice a week. A second evaluation will be performed during the sixth session treatment with algometry, electromyographic biofeedback and visual analog pain scale, before IC application. The last VG evaluation will be performed one day after the 10th session, with algometry, electromyographic biofeedback and analogue visual scale for pain. The GWV will be evaluated in a single moment with algometry, electromyographic biofeedback and visual analogue scale for pain. This group will be evaluated just one time in order to obtain a reference of pelvic floor musculature without sexual dysfunction. 


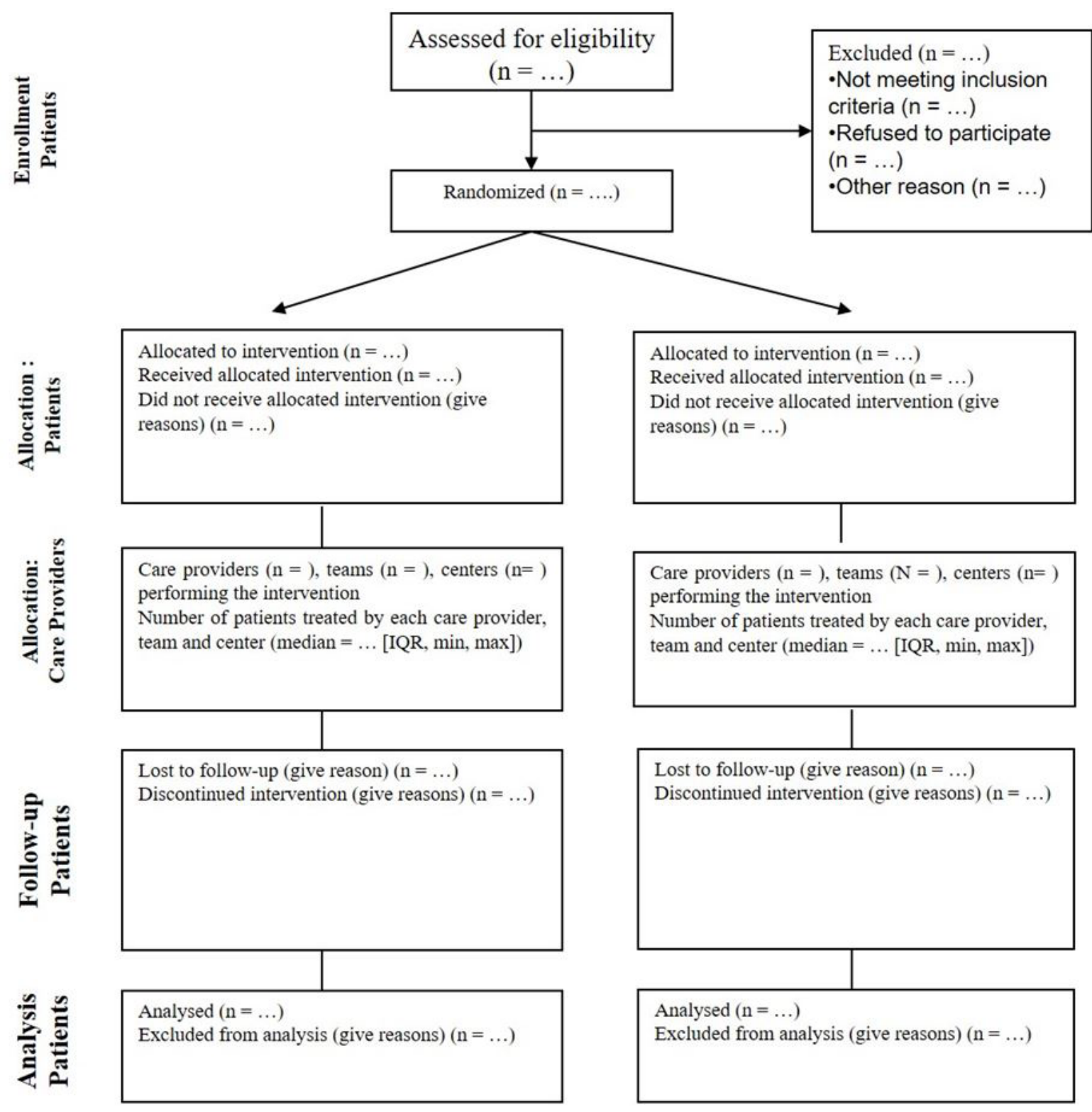

Figure 1. Diagram representing the flow of participants at each stage of the study.

\section{Protocol}

After completing the Informed Consent Form, all participants will be submitted to anamnesis, composed of demographic data, sexual, gynecological, proctological and urological history. After anamnesis the participant will be submitted to the physical examination in gynecological position, there will be the inspection and palpation of the perineum with the observation of muscular trophism, coloration, alterations of skin of the vulva, perineum and anus. The palpation will be performed with one finger in the perineum region to verify the muscle tone, voluntary contraction will be requested.

The pain threshold in the perineum region will be evaluated with a pressure algometer. The participant will be placed in the supine position, with the pelvis in a neutral position, hip and knee flexion and feet support on the stretcher. The algometer will be pressed progressively and gradually, at a rate of $1 \mathrm{~kg} / \mathrm{sec}$, at four points, one at the central tendon of perineum and three at the vaginal opening - $6 \mathrm{hs}, 4 \mathrm{hs}$ and $8 \mathrm{hs}$ according to the imaginary clock in the vaginal introitus ${ }^{21,22}$, according Figure 2 .

Pain will also be evaluated with the help of VAS ${ }^{10}$. The scale will be shown to the study participant and she will have to refer / point / indicate her pain during intercourse or attempted intercourse according to the scale. The VAS score ranges from zero to 10 and the higher the score, the greater the intensity of the pain. The VAS ${ }^{10}$ also subclassifies the light intensity of pain with a score from zero to two, moderate from three to seven and intense from eight to 10 . The muscular electric activity 


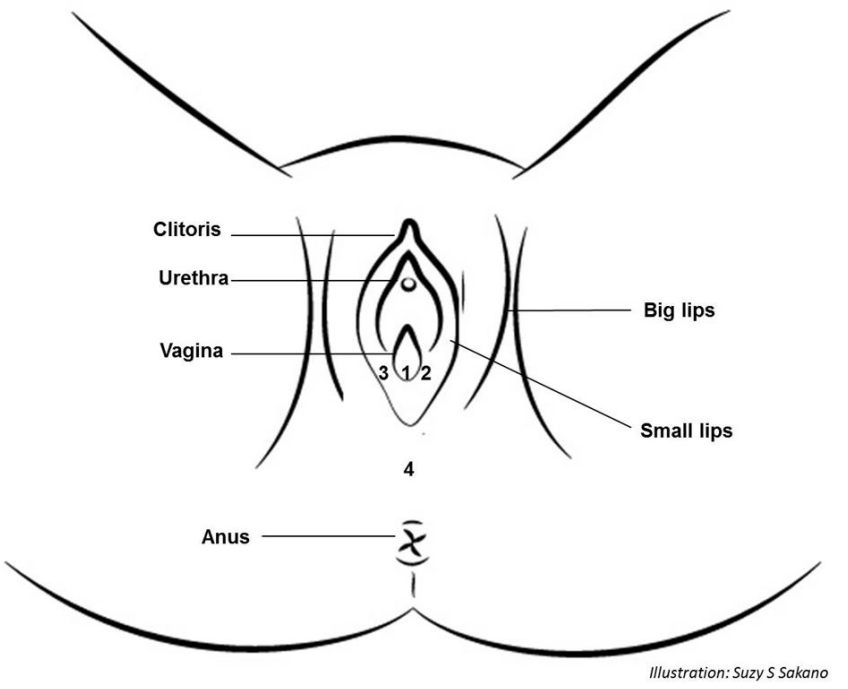

Figure 2. Illustrative image with the identification of the points evaluated with the algometer. Legend: $1=6 \mathrm{hs} ; 2=4 \mathrm{hs} ; 3=8 \mathrm{hs} ; 4=$ central tendon of perineum. Source: Ambulatory of Pelvic Physical Therapy of Santa Casa de São Paulo.
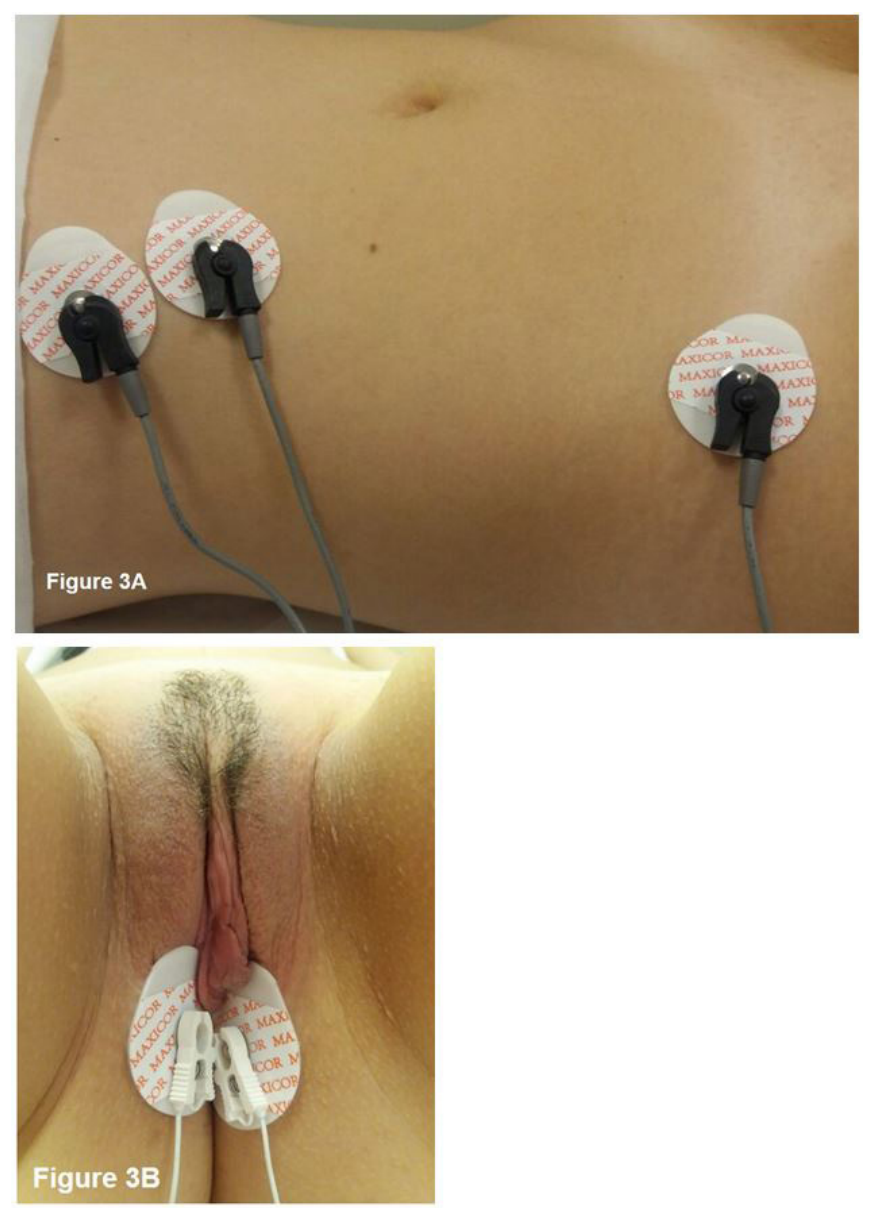

Figure 3A. Illustrative image of the positioning of the abdominal sensors in the right internal oblique muscle and in the anterior superior iliac crest. (B) Positioning of the two surface electrodes in the perineum region used in the evaluation and reassessment of VG and GWV. Source: Ambulatory of Pelvic Physical Therapy of Santa Casa de São Paulo.

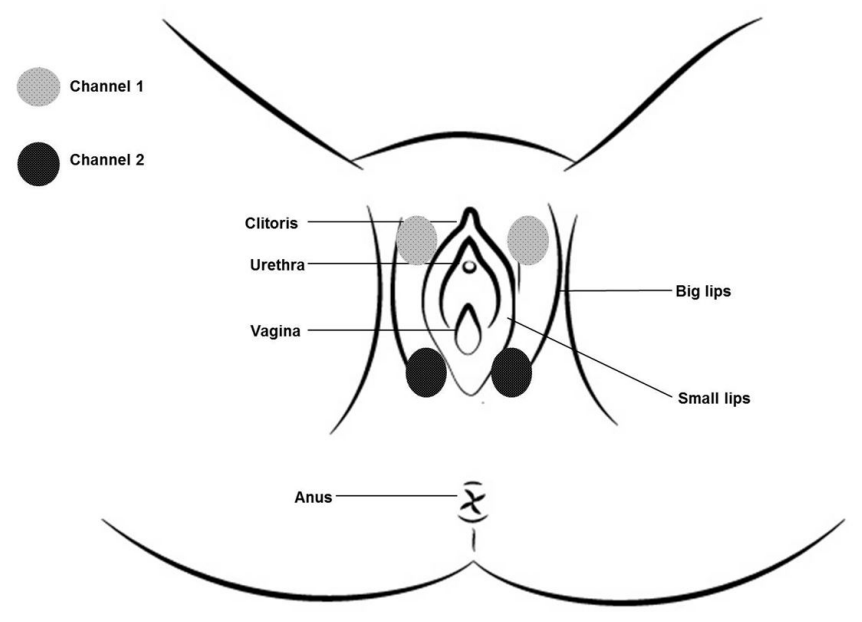

Figure 4. Illustrative image of the positioning of the electrodes used in the 10 sessions of application of the interferential current in the vaginismus group.

will be evaluated by the electromyographic biofeedback, with the participant lying in the supine position, pelvis in a neutral position, hip and knee flexion and feet support on the stretcher. Two abdominal sensors will be placed on the right internal oblique muscle and a surface electrode on the anterior superior iliac crest on the same side to capture the electrical activity of the abdominal muscles (Fig. 3A). Two surface electrodes will be placed in the perineum region to capture the electrical activity of the perineal muscles (Fig. 3B).

\section{Intervention}

The electrostimulation will be performed in 10 sessions, with the use of IC, the carrier frequency will be $2000 \mathrm{~Hz}$, with modulated amplitude of frequency of $80 \mathrm{~Hz}$, pulse width of $200 \mu$ s and the intensity being modulated according to the sensory level of each participant. The position of the electrodes will be maintained in all sessions according to Fig. 4 and the application of the current will last 30 minutes, twice a week ${ }^{15}$.

\section{Statistical Analysis}

After the protocol application, statistical analysis will be performed. The data of the VG before and after the protocol application will be compared. The level of significance will be the value of $p<0.05$.

\section{Trial Status}

Participants are being recruited at the time of submission.

\section{AUTHORS' CONTRIBUTIONS:}

CMAP and RTPA elaborated the study design; CMAP performed the data collection; EMCMB, SMRRL and VLSA performed the critical intellectual revision of the manuscript. All authors read and approved the final manuscript;

\section{CONFLICTS OF INTEREST:}

nothing to declare. 


\section{AUTHORS DETAILS}

${ }^{2}$ Anhanguera University and University City de São Paulo - São Paulo - SP Brasil. ${ }^{3}$ Mantelli Clinic - São Paulo - SP - Brasil

\section{REFERENCES}

1. Abdo $\mathrm{CHN}$, Oliveira WM. O ginecologista brasileiro frente às queixas sexuais femininas: um estudo preliminar. Rev Bras Med. 2002; 59 (3):179-86.

2. Abdo $\mathrm{CHN}$, Fleury HJ. Aspectos diagnósticos e terapêuticos das disfunções sexuais femininas. Rev Psiq Clín. 2006; 33 (3):162-7.

3. Segraves $\mathrm{R}$, Balon R, Clayton A. Proposal for changes in diagnostic criteria for sexual dysfunction. J Sex Med. 2007; 4: 567-80.

4. Bortolami A, Vanti C, Banchelli F, Guccione AA, Pillastrini P. Relationshop between female pelvic floor dysfunction and sexual dysfunction: $\mathrm{Na}$ observational study. J Sex Med. 2015; 12: 1233-41.

5. Lahaie MA, Amsel R, Khalife S, Boyer S, Faaborg-Andersen M, Binik YM. Can Fear, Pain, and Muscle Tension Discriminate Vaginismus from Dyspareunia/Provoked Vestibulodynia? Implications for the New DSM-5 Diagnosis of Genito-Pelvic Pain/Penetration Disorder. Archives of sexual behavior. 2015; 44 (6):1537-50.

6. American Psychiatric Association. Diagnostic and statistical manual of mental disorders. Fifth edition - DSM- $5^{\mathrm{TH}}$. Arlignton, VA. American Psychiatric Association, 2013.

7. Antonioli R.S., Simões D. Abordagem fisioterapêutica nas disfunções sexuais femininas. Rev Neurocienc. 2010; 18 (2): 267-74.

8. Pérez, MCN. Colégio Mexicano de especialistas em ginecologia e obstetrícia. 2010; 78 (4): 532-34.

9. Brosens C, Terrasa S, Astolfi E. Actualización: Vaginismo. Actualización en la Práctica Ambulatoria. 2009; 12 (3): 102-103.

10. Agne, J.E. Eletrotermoterapia, Teoria e Prática. Editora Orium, 1aed Santa Maria SC: Pallotti, 2004;

11. Hilgenberg-Sydney PB, Kowacs PA, Conti, PCR. Somatosensory Evaluation in Dysfunctional Syndrome Patients. Journal of Oral Rehabilitation. 2016; 43: 89-95.

12. Noronha J, Pereira SB. Eletromiografia aplicada ao assoalho pélvico. In: Urofisioterapia Aplicações clínicas das técnicas fisioterapêuticas nas disfunções miccionais e do assoalho pélvico: Paulo César Rodrigues Palma. São Paulo: 2014; 142-148.
13. Herbenick D, Schick V, Sanders AS, Reece M, Fortenberry D. Pain experienced during vaginal and anal intercourse with other-sex partners: Findings from nationally representative probability study in the United States. J Sex Med. 2015; (12): 1040-51.

14. Artioli DP, Bertolini GRF. Interferential current: application, parameters and results. Rev Bras Clin Med. 2012; 10 (1): 51-6.

15. Yaraghi M, Ghazizadeh S, Mohammadi F, Ashtiani EM, Bakhtiyari M, Mareshi SM et al. Comparing the effectiveness of functional electrical stimulation via sexual cognitive/behavioral therapy of pelvic floor muscles versus local injection of botulinum toxin on the sexual functioning of patients with primary vaginismus: a randomized clinical trial. Int Urogynecol J. 2019 Nov; 30 (11): 1821-28.

16. Faccil LM, Nowotnyl JP, TormemI F, Trevisanil VFM. Effects of transcutaneous electrical nerve stimulation (TENS) and interferential currents (IFC) in patients with nonspecific chronic low back pain: randomized clinical trial. Sao Paulo Med J. 2011; 129 (4): 206-16.

17. Dohnert MB, Bauer JP, Pavão TS. Study of the effectiveness of interferential current as compared to transcutaneous electrical nerve stimulation in reducing chronic low. Rev Dor. 2015; 16 (1): 27-31.

18. Rosenbaum TY. Addressing anxiety in vivo in physiotherapy treatment of women with severe vaginismus: a clinical approach. J Sex Marital Ther. 2011; 37: 89-93.

19. Reissing ED, Armstrong $\mathrm{HL}$, Allen C. Pelvic Floor Physical Therapy for Lifelong Vaginismus: A Retrospective Chart Review and Interview Study. J Sex Marital Ther. 2013; 39: 306-20.

20. Bortolam A, Vanti, C, Banchelli F et al. Relationship Between Female Pelvic Floor Dysfunction and Sexual Dysfunction: An Observational Study. J Sex Med. 2015; 12: 1233-41.

21. Pacik PT. Understanding and treating vaginismus: a multimodal approach. Int Urogynecol J. 2014; 25: 1613-20.

22. Kinser AM, Sands WA, Stone MH. Reliability and validity of a pressure algometer. J Strength Cond Res. 2009; 23 (1): 312-4.

23. Organização Mundial da Saúde. CID-11 Classificação Estatística Internacional de Doenças e Problemas Relacionados à Saúde. 11a rev. São Paulo: Universidade de São Paulo; 2018.

24. Maciel LFM, Ferreira JJA, Santos HH, Andrade PR. Efeitos da Estimulação Elétrica Nervosa Transcutânea e da Crioterapia sobre o Limiar de Dor Induzida por Pressão. Fisioter Pesq. 2014; 21 (3): 249-56. 